\title{
Effect of In Vitro Testing Parameters on Ceftazidime-Avibactam Minimum Inhibitory Concentrations
}

\author{
Tiffany R. Keepers, Marcela Gomez, Donald Biek, Ian Critchley, and Kevin M. Krause \\ Cerexa, Inc., Oakland, CA 94612, USA \\ Correspondence should be addressed to Tiffany R. Keepers; tkeepers@gmail.com
}

Received 13 February 2015; Accepted 16 April 2015

Academic Editor: Wladimir Sougakoff

Copyright ( 2015 Tiffany R. Keepers et al. This is an open access article distributed under the Creative Commons Attribution License, which permits unrestricted use, distribution, and reproduction in any medium, provided the original work is properly cited.

Effects of varying in vitro susceptibility testing parameters of the broth microdilution assay on ceftazidime-avibactam MICs were determined and compared to meropenem and piperacillin-tazobactam for 9 Enterobacteriaceae and 4 Pseudomonas aeruginosa isolates. The effect of varying incubation conditions (ambient air or $5 \% \mathrm{CO}_{2}$ ), $\mathrm{pH}$ of medium, medium composition (cation-adjusted Mueller Hinton Broth with and without laked horse blood and Haemophilus Test Medium), cation content of the medium, and inoculum density were tested. Most variations had no effect on ceftazidime-avibactam MIC values (no more than a 2-fold change). However, acidic $\mathrm{pH}$ or high inoculum resulted in 4- to 16-fold changes in MIC, which was similar to those observed for meropenem and piperacillin-tazobactam under these conditions. Overall, this study shows that slight variations in testing parameters during routine MIC testing will likely have no significant effect on ceftazidime-avibactam MIC values.

\section{Introduction}

Avibactam is a non- $\beta$-lactam- $\beta$-lactamase inhibitor with activity against class $\mathrm{C}$ enzymes, most class $\mathrm{A}$ enzymes (including KPCs), and some class D (OXA) carbapenemases [1]. When ceftazidime is combined with avibactam, the spectrum of activity is expanded to include organisms producing these $\beta$-lactamases [2-4]. This combination is currently in Phase 3 clinical trials for the treatment of Gram-negative infections.

Susceptibility testing conditions can vary slightly between laboratories and between laboratory scientists. It is important to know if small variations from standard Clinical Laboratory Standards Institute (CLSI) testing guidelines cause any significant changes in testing outcome. These variations can have effects on the MIC when testing $\beta$-lactams, including cephalosporins and $\beta$-lactam- $\beta$-lactamase inhibitor combinations [5-7]. The effect of varying the broth microdilution in vitro testing parameters on the ceftazidime-avibactam MIC of $\beta$-lactamase positive and negative Enterobacteriaceae and Pseudomonas aeruginosa isolates was determined and compared with the effects on meropenem and piperacillintazobactam MICs.

\section{Materials and Methods}

Isolates were obtained from ATCC (Manassas, VA), JMI Laboratories (North Liberty, IA), or the Cerexa, Inc. culture collection. MICs were determined for ceftazidimeavibactam (ceftazidime: USP, avibactam: Forest Laboratories, Inc.), meropenem (USP), and piperacillin-tazobactam (piperacillin: Sigma, tazobactam: USP) by broth microdilution assay according to CLSI guidelines $[8,9]$. The effects of the following parameters on the MIC were determined:

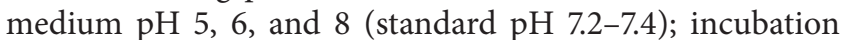
with $5 \% \mathrm{CO}_{2}$ (standard incubation ambient air); the addition of 2.5\% laked horse blood (LHB) to cation-adjusted Mueller Hinton broth (CAMHB) and testing in Haemophilus Test Medium (HTM) (standard medium CAMHB); testing in non-cation-adjusted media (MHB without added cations containing $2.9-5.9 \mathrm{mg} / \mathrm{L}$ calcium and $3.2-5.2 \mathrm{mg} / \mathrm{L}$ magnesium) and testing in $50 \mathrm{mg} / \mathrm{L}$ calcium in MHB (standard cation concentration $20-25 \mathrm{mg} / \mathrm{L}$ calcium and $10-12.5 \mathrm{mg} / \mathrm{L}$ magnesium); and inoculum densities of $5 \times 10^{4} \mathrm{CFU} / \mathrm{mL}$ and $5 \times 10^{6} \mathrm{CFU} / \mathrm{mL}$ (standard density $5 \times 10^{5} \mathrm{CFU} / \mathrm{mL}$ ). MIC tests were performed in 2 separate experiments. Data tables list the highest MIC value recorded. Human serum 


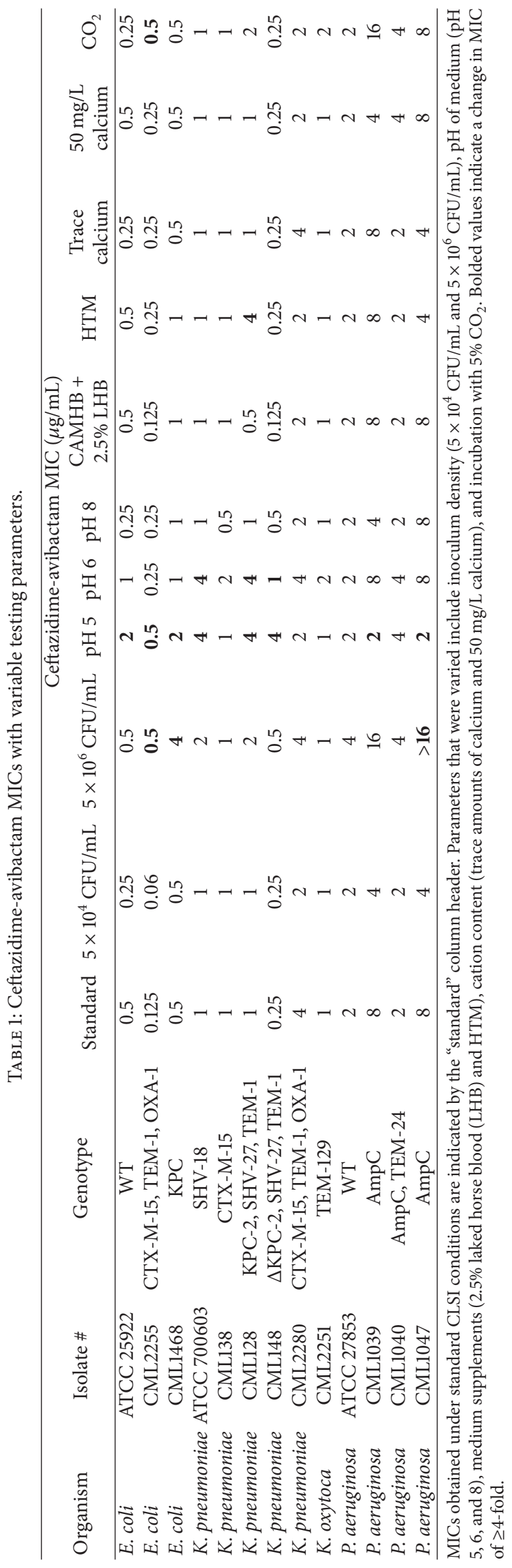




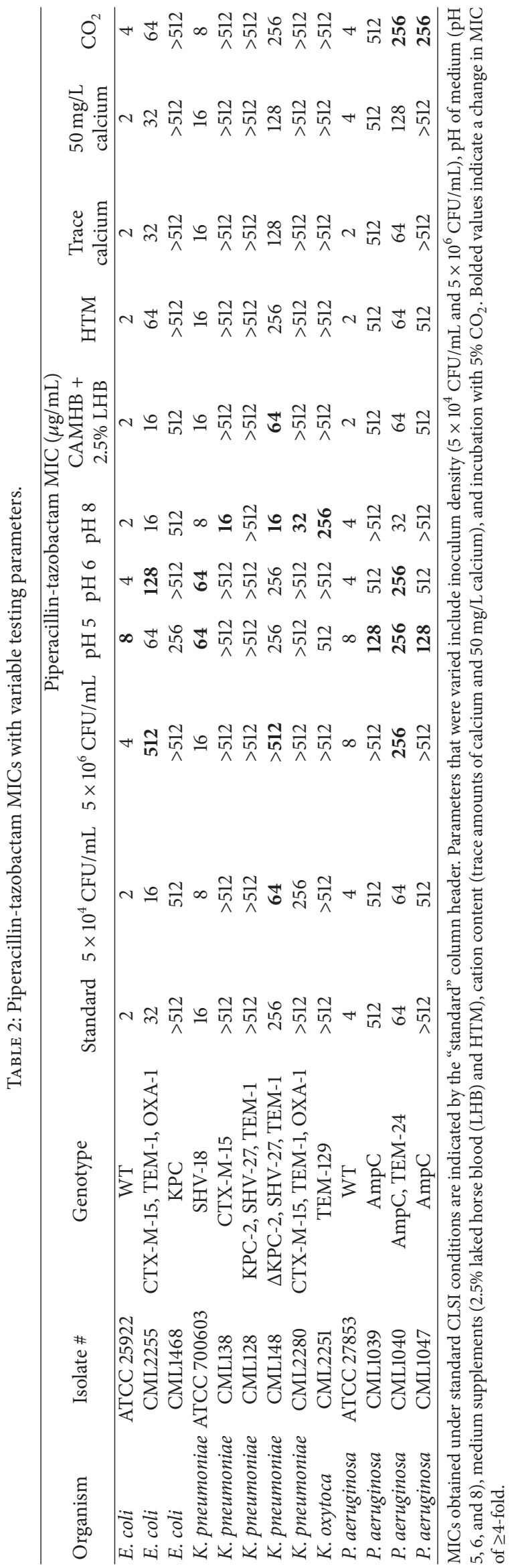




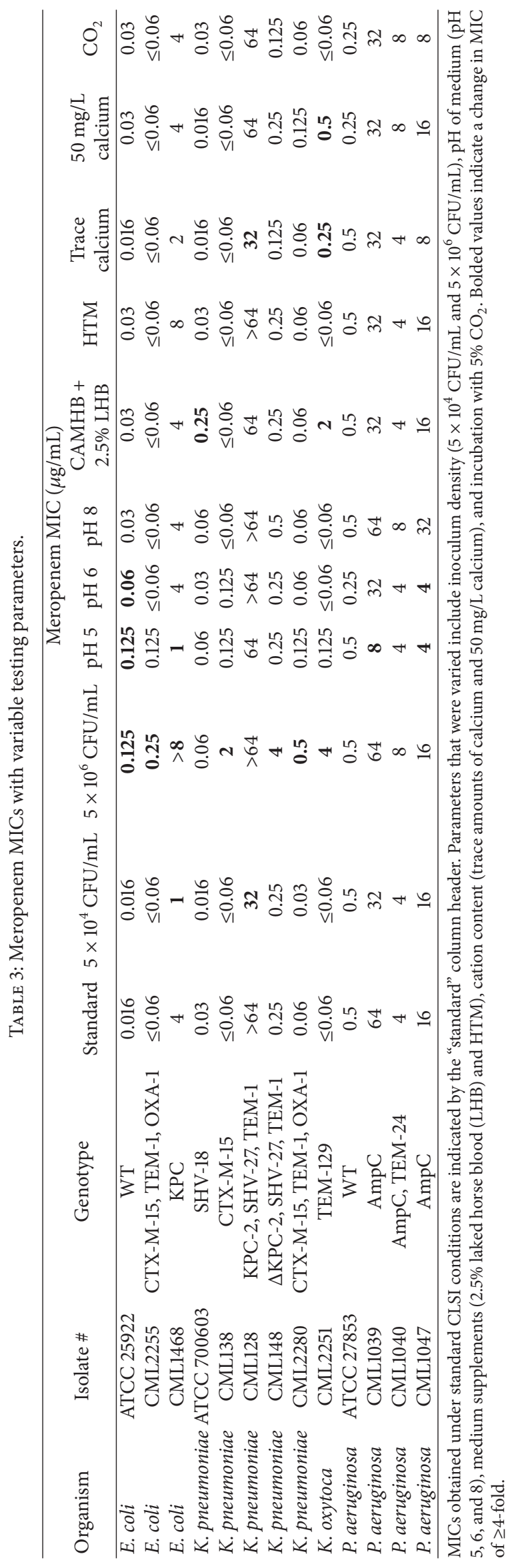


and human serum albumin were previously shown to have no effect on ceftazidime-avibactam MICs [10].

\section{Results and Discussion}

3.1. Media $p H$. In medium with acidic $\mathrm{pH}(\mathrm{pH} 5$ or 6$)$, MICs for ceftazidime-avibactam were increased by 4 - to 16-fold for 6 of 9 Enterobacteriaceae isolates and were decreased by 4 -fold for 2 of 4 P. aeruginosa isolates (Table 1). Acidic media caused increased piperacillin-tazobactam MICs against 4 isolates and decreased MICs against 3 isolates by $\geq 4$-fold (Table 2). Four isolates had 4 - to $>32$-fold decreased piperacillin-tazobactam MICs when tested using medium at $\mathrm{pH}$ 8. Acidic media caused increased meropenem MICs against 4 isolates and decreased meropenem MICs against 3 isolates by 4 - to 8 -fold (Table 3 ). MIC increases were observed at $\mathrm{pH} 5$ against the reference strain E. coli ATCC $25922(\beta-$ lactamase negative). Thus, the $\mathrm{pH}$ effect on MIC appears to be related to the intrinsic antibacterial activity of the $\beta$-lactams, rather than having an effect on the inhibition of $\beta$-lactamases.

3.2. Incubation Conditions. The ceftazidime-avibactam MIC for one $E$. coli isolate was increased by 4 -fold when incubated in $5 \% \mathrm{CO}_{2}$ (Table 1). Incubation in $5 \% \mathrm{CO}_{2}$ caused the piperacillin-tazobactam MICs against $2 P$. aeruginosa isolates to change by $\geq 4$-fold (Table 2 ). The meropenem MICs for all isolates were unaffected (Table 3 ).

3.3. Media Composition. Testing in HTM had no effect on MICs with any drug except for one isolate that had 4-fold increased ceftazidime-avibactam MIC (Table 1). Addition of $2.5 \%$ LHB had no effect on ceftazidime-avibactam MICs (Table 1), caused a 4-fold decreased piperacillin-tazobactam MIC against one isolate (Table 2 ), and caused 8 -fold and $>32$ fold increased meropenem MICs for 2 isolates (Table 3).

3.4. Calcium Content. Ceftazidime-avibactam and piperacillin-tazobactam MICs were unaffected by changes in calcium concentration of media (Tables 1 and 2 ). In medium with trace calcium (MHB), MICs of meropenem decreased by $\geq 4$-fold against one isolate and increased by $>4$-fold against another (Table 3).

3.5. Inoculum. Three isolates had ceftazidime-avibactam MICs that were increased by 4 - to 8 -fold when the inoculum was increased 10 -fold $\left(5 \times 10^{6} \mathrm{CFU} / \mathrm{mL}\right)$ (Table 1$)$. Increased inoculum raised the MICs 4- to 16 -fold for piperacillintazobactam against 3 isolates, and lower inoculum $(5 \times$ $10^{4} \mathrm{CFU} / \mathrm{mL}$ ) resulted in a 4 -fold decreased MIC for one isolate (Table 2). High inoculum density caused meropenem MICs to increase by 4 - to $\geq 64$-fold for 7 isolates, while low inoculum density caused meropenem MICs to decrease by 4- to 8 -fold for 2 isolates (Table 3).

\section{Conclusions}

In summary, most of the variations in broth microdilution parameters had little effect on ceftazidime-avibactam MICs. The two parameters that did have an effect were acidic media
(6 isolates) and high inoculum density (3 isolates). Changes in MIC for these conditions ranged from 4- to 16-fold. Other changes to testing parameters only affected ceftazidimeavibactam MICs for a single isolate by only 4-fold in each instance. It is possible that these individual cases are due to random variation rather than reproducible effects on MIC. Piperacillin-tazobactam and meropenem MICs were also affected by changes in $\mathrm{pH}$ and inoculum density indicating that these effects are not unique to the ceftazidime-avibactam combination. The magnitude of the changes in piperacillintazobactam MICs was more difficult to assess due to the high MICs of most isolates under standard testing conditions.

The inoculum effects noted in this study with ceftazidime-avibactam MICs are not surprising given that inoculum effects have been previously documented with ceftazidime MIC testing, particularly against $\beta$-lactamase producing strains [5-7]. An inoculum effect on other cephalosporin and carbapenem MICs has also been previously documented; several studies have shown that MICs for piperacillintazobactam, and to a lesser extent meropenem, are increased with higher inocula of $\beta$-lactamase producing and nonproducing isolates $[6,7,11-13]$.

These findings indicate that slight variations in testing parameters during routine MIC testing will likely have no significant effect on ceftazidime-avibactam MIC values; however, special attention should be paid to closely follow CLSI guidelines with respect to the $\mathrm{pH}$ of the media and the inoculum used. Since most laboratories use commercially prepared media, $\mathrm{pH}$ should not be an issue due to the buffering capacity of these media. Additionally, CLSI recommends validating inoculum density on a weekly basis as part of standard quality control so any variations in inoculum should be easily identified [8]. Therefore, if CLSI guidelines and quality control recommendations are followed, there should not be any issues with routine ceftazidime-avibactam MIC testing.

\section{Disclosure}

The authors were employees of Cerexa at the time the study was conducted.

\section{Conflict of Interests}

The authors declare that there is no conflict of interests regarding the publication of this paper.

\section{Acknowledgments}

This research was funded by Forest Laboratories LLC, a wholly owned indirect subsidiary of Actavis plc, and Cerexa, Inc., a wholly owned subsidiary of Forest Laboratories. Cerexa, Inc. was involved in the design, collection, analysis, and interpretation of data and the decision to present these results.

\section{References}

[1] P. Lagacé-Wiens, A. Walkty, and J. A. Karlowsky, "Ceftazidimeavibactam: an evidence-based review of its pharmacology and potential use in the treatment of Gram-negative bacterial infections," Core Evidence, vol. 9, pp. 13-25, 2014. 
[2] M. Castanheira, S. E. Farrell, K. M. Krause, R. N. Jones, and H. S. Sader, "Contemporary diversity of $\beta$-lactamases among enterobacteriaceae in the nine U.S. census regions and ceftazidime-avibactam activity tested against isolates producing the most prevalent $\beta$-lactamase groups," Antimicrobial Agents and Chemotherapy, vol. 58, no. 2, pp. 833-838, 2014.

[3] R. K. Flamm, G. G. Stone, H. S. Sader, R. N. Jones, and W. W. Nichols, "Avibactam reverts the ceftazidime MIC90 of European Gram-negative bacterial clinical isolates to the epidemiological cut-off value," Journal of Chemotherapy, vol. 26, no. 6, pp. 333-336, 2014.

[4] H. S. Sader, M. Castanheira, R. K. Flamm, D. J. Farrell, and R. N. Jones, "Antimicrobial activity of ceftazidime-avibactam against gram-negative organisms collected from U.S. medical centers in 2012," Antimicrobial Agents and Chemotherapy, vol. 58, no. 3, pp. 1684-1692, 2014.

[5] C.-I. Kang, M. K. Cha, S. H. Kim et al., "Extended-spectrum cephalosporins and the inoculum effect in tests with CTX-Mtype extended-spectrum $\beta$-lactamase-producing Escherichia coli: potential clinical implications of the revised CLSI interpretive criteria," International Journal of Antimicrobial Agents, vol. 43, no. 5, pp. 456-459, 2014.

[6] B. Segatore, D. Setacci, M. Perilli et al., "Antimicrobial susceptibility of clinical isolates of Enterobacteriaceae producing complex $\beta$-lactamase patterns including extended-spectrum enzymes," International Journal of Antimicrobial Agents, vol. 23, no. 5, pp. 480-486, 2004.

[7] K. S. Thomson and E. S. Moland, "Cefepime, piperacillintazobactam, and the inoculum effect in tests with extendedspectrum beta-lactamase-producing Enterobacteriaceae," Antimicrobial Agents and Chemotherapy, vol. 45, no. 12, pp. 3548-3554, 2001.

[8] CLSI, "Performance standards for antimicrobial susceptibility testing; twenty-third informational supplement," CLSI Document M100-S23, Clinical and Laboratory Standards Institute, Wayne, Pa, USA, 2013.

[9] CLSI, Methods for Dilution Antimicrobial Susceptibility Tests for Bacteria That Grow Aerobically; Approved Standard, CLSI Document M07-A9, Clinical and Laboratory Standards Institute, Wayne, Pa, USA, 9th edition, 2012.

[10] T. R. Keepers, M. Gomez, C. Celeri, W. W. Nichols, and K. M. Krause, "Bactericidal activity, absence of serum effect, and timekill kinetics of ceftazidime-avibactam against $\beta$-lactamaseproducing enterobacteriaceae and Pseudomonas aeruginosa," Antimicrobial Agents and Chemotherapy, vol. 58, no. 9, pp. 52975305, 2014.

[11] Y. Harada, Y. Morinaga, N. Kaku et al., "In vitro and in vivo activities of piperacillin-tazobactam and meropenem at different inoculum sizes of ESBL-producing Klebsiella pneumoniae," Clinical Microbiology and Infection, vol. 20, no. 11, pp. O831O839, 2014.

[12] L. López-Cerero, E. Picón, C. Morillo et al., "Comparative assessment of inoculum effects on the antimicrobial activity of amoxycillin-clavulanate and piperacillin-tazobactam with extended-spectrum $\beta$-lactamase-producing and extendedspectrum $\beta$-lactamase-non-producing Escherichia coli isolates," Clinical Microbiology and Infection, vol. 16, no. 2, pp. 132-136, 2010.

[13] V. H. Tam, K. R. Ledesma, K. T. Chang, T. Y. Wang, and J. P. Quinn, "Killing of Escherichia coli by $\beta$-lactams at different inocula," Diagnostic Microbiology and Infectious Disease, vol. 64, no. 2, pp. 166-171, 2009. 

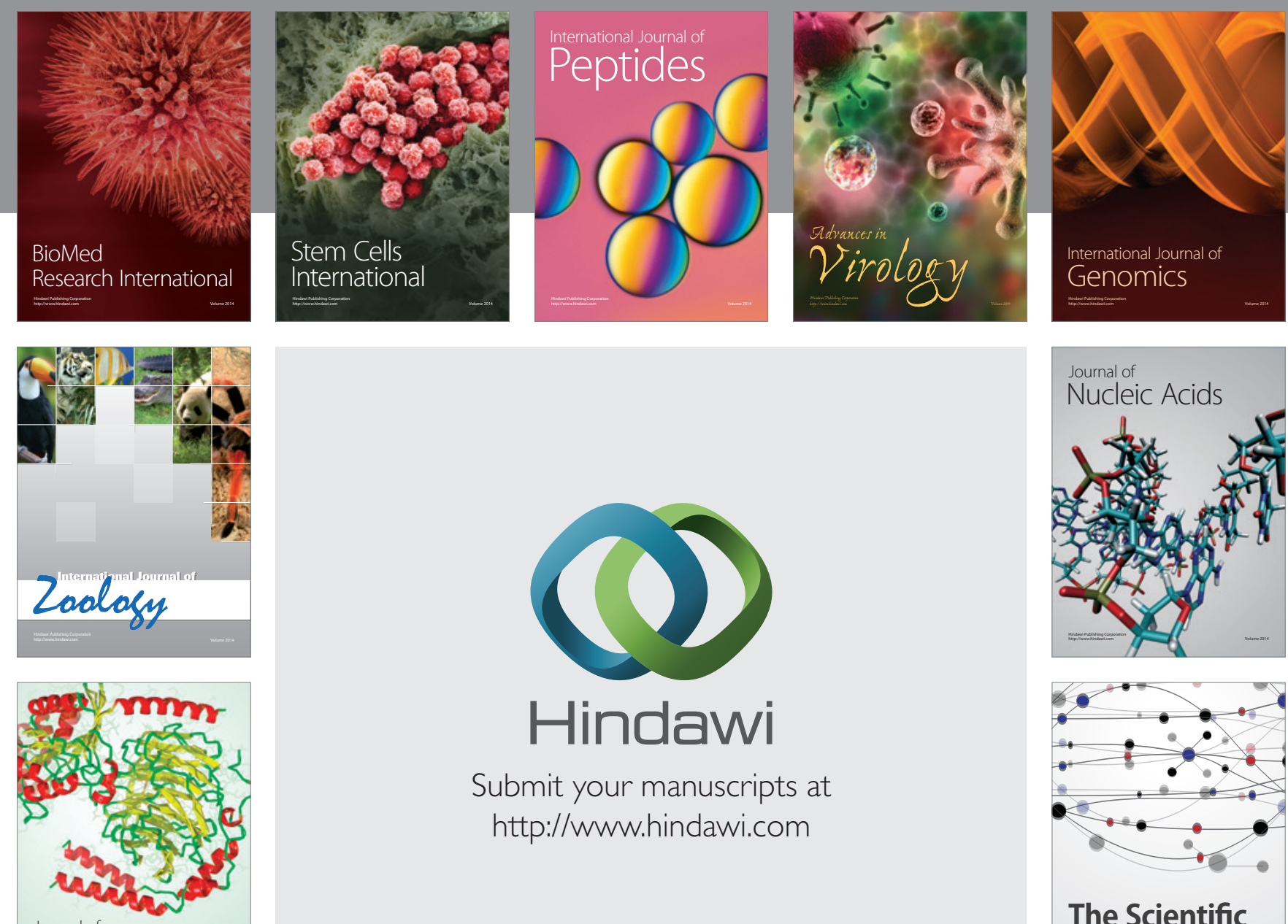

Submit your manuscripts at

http://www.hindawi.com

Journal of
Signal Transduction
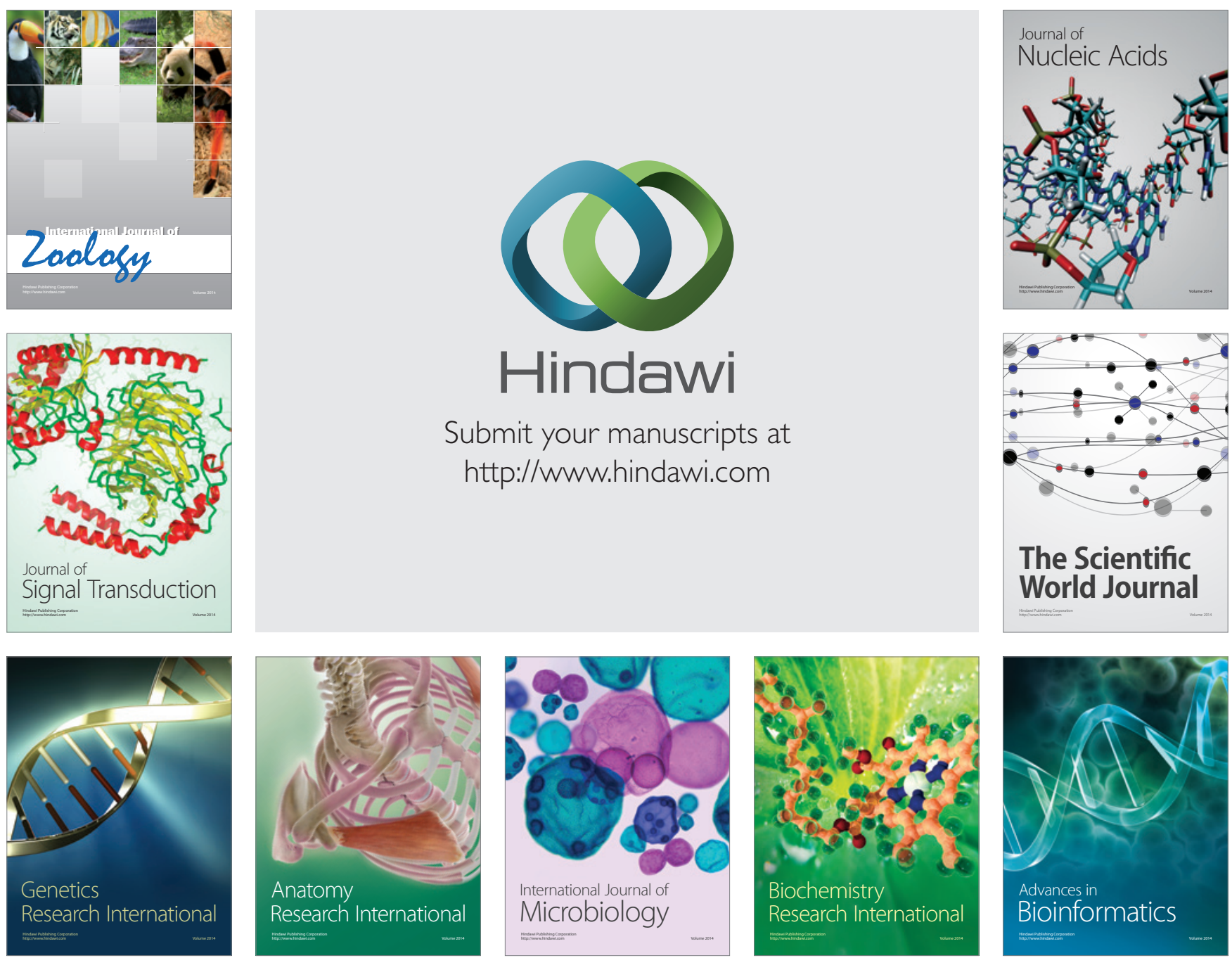

The Scientific World Journal
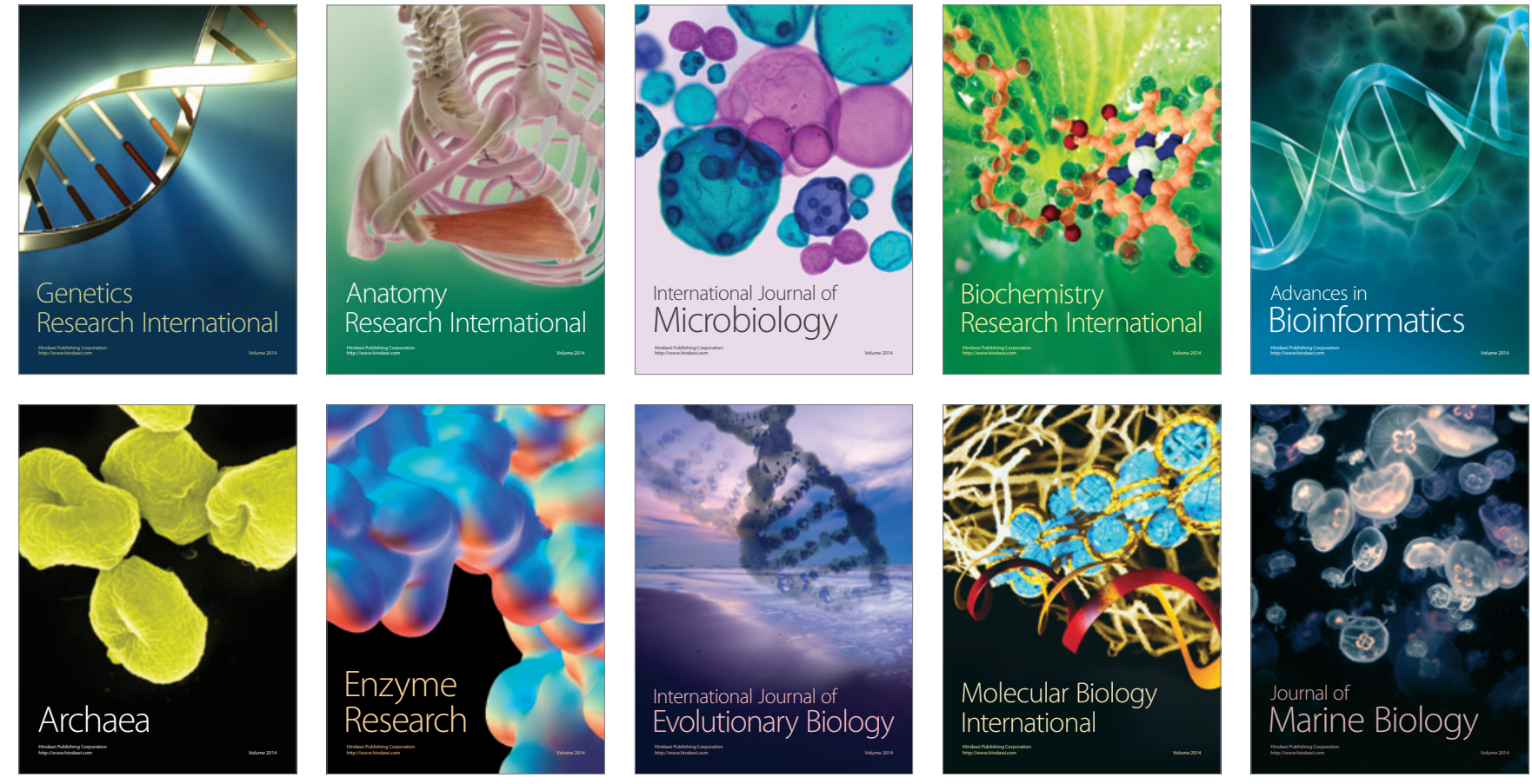